DOC.

D101.60/5:

2000-01

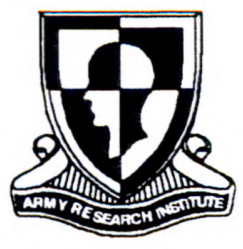

Research Product 2000-01

\title{
A Review and Annotated Bibliography of the Literature Pertaining to Team and Small Group Performance (1989 to 1999)
}

December 1999

\section{Armored Forces Research Unit}

U.S. Army Research Institute for the Behavioral and Social Sciences 